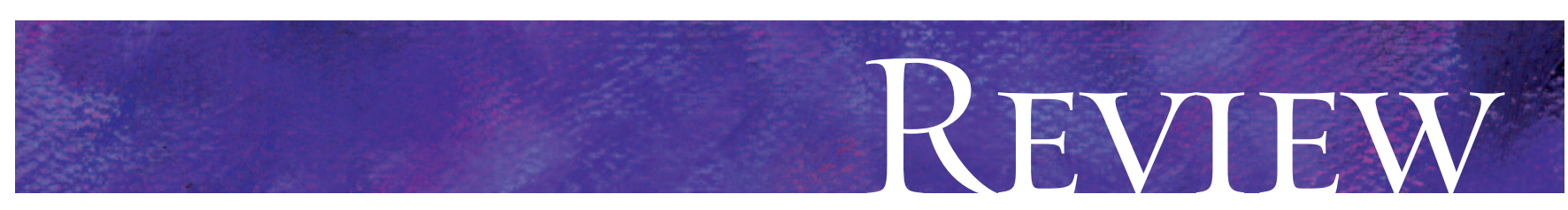

\title{
Management of acute decompensated heart failure
}

\author{
Larry A. Allen, Christopher M. O’Connor
}

\section{ABSTRACT}

Acute decompensated heart failure represents a heterogeneous group of disorders that typically present as dyspnea, edema and fatigue. Despite the high prevalence of this condition and its associated major morbidity and mortality, diagnosis can be difficult, and optimal treatment remains poorly defined. Identification of the acute triggers for the decompensation as well as noninvasive characterization of cardiac filling pressures and output is central to management. Diuretics, vasodilators, continuous positive airway pressure and inotropes can be used to alleviate symptoms. However, few agents currently available for the treatment of acute decompensated heart failure have been definitively shown in large prospective randomized clinical trials to provide meaningful improvements in intermediate-term clinical outcomes. Multiple novel therapies are being developed, but previous treatment failures indicate that progress in the management of acute decompensated heart failure is likely to be slow.

CMAJ 2007;176(6):797-805

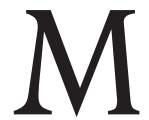
ost experienced practitioners are familiar with the patient who presents with shortness of breath from an exacerbation of congestive heart failure (also known as acute decompensated heart failure). However, options for the management of these patients remain crude and limited. ${ }^{1,2}$ Acute decompensated heart failure is a common and growing medical problem associated with major morbidity and mortality. ${ }^{3-6}$ It is the leading reason for hospital admission among patients over age 65 and the most costly cardiovascular disorder in Western countries. The 6o-day mortality following hospital admission because of an exacerbation of congestive heart failure is $8 \%-20 \%$, depending on the population studied. ${ }^{8,9}$

The difficulties surrounding treatment begin with a lack of clear definitions. The term "acute decompensated heart failure" broadly represents new or worsening symptoms or signs of dyspnea, fatigue or edema that lead to hospital admission or unscheduled medical care and that are consistent with an underlying worsening of left ventricular function. ${ }^{10}$ Acute heart failure defined as the onset of symptoms or signs of heart failure in a patient with no prior history of heart failure and previously normal function is an uncommon cause of acute decompensated heart failure, particularly in patients without concomitant acute coronary syndromes. Much more frequently, acute decompensated heart failure occurs in pa- tients with previously established myocardial dysfunction (systolic or diastolic) who present with an exacerbation of symptoms or signs after a period of relative stability. Acute decompensated heart failure represents a heterogeneous group of disorders with various causes (Box I), many of which are not yet well understood. ${ }^{11}$ Recent large registries of patients with exacerbation of congestive heart failure show that about $50 \%$ of them have preserved systolic function (left ventricular ejection fraction $>50 \%$ ). ${ }^{12}$ Because patients with underlying chronic diastolic dysfunction seem to have a narrow window for optimal volume status, and because so few targeted therapies are available for diastolic dysfunction, acute decompensated heart failure in this subset of patients presents a unique challenge to clinicians.

With a handful of randomized trials over the past 5 years investigating new treatments of acute decompensated heart failure, the field has recently received increasing attention. Here, we attempt to briefly examine the physiologic rationale for the currently available treatments and summarize the existing evidence for their efficacy, with the goal of informing providers how to optimize their management of patients with acute decompensated heart failure. Our review is meant to complement the more comprehensive guidelines for the diagnosis and treatment of acute heart failure issued by the European Society of Cardiology ${ }^{1}$ and the Heart Failure Society of America. $^{2}$

\section{Diagnosis}

The rapid diagnosis of acute decompensated heart failure is necessary to initiate appropriate treatment. Failure to do so increases the need for mechanical ventilatory support, delays hospital discharge and inflates treatment costs. ${ }^{13,14}$ Unfortunately, the signs and symptoms of acute decompensated heart failure often overlap with those of other common medical conditions, particularly chronic obstructive pulmonary disease. In addition, because of the heterogeneous nature of acute decompensated heart failure, no single finding is perfect for diagnosis, and instead, a broad array of signs and symptoms are associated with the condition (Box 2). ${ }^{15}$ Of the associated symptoms, dyspnea on exertion is the most sensitive (negative likelihood ratio $0.45,95 \%$ confidence interval [CI] $0.35-0.67$ ), whereas paroxysmal nocturnal dyspnea is the most specific (positive likelihood ratio $2.6,95 \%$ CI I.54.5).$^{16}$ On physical examination, elevated jugular venous pressure is the best indicator for identifying acute decompensated heart failure (positive likelihood ratio 5.I, 95\% CI 3.2-7.9; 
negative likelihood ratio $0.66,95 \% \mathrm{CI} 0.57-0.77$ ), although measurement of jugular venous pressure by clinicians is notoriously inaccurate. ${ }^{16}$

A number of tests can be performed to help with the diagnosis (Box 3). The presence of pulmonary venous congestion and interstitial edema on chest radiographs increases the likelihood of acute decompensated heart failure about I2fold. ${ }^{16} \mathrm{~A}$ low level of $\mathrm{B}$-type natriuretic peptide or $\mathrm{N}$-terminal B-type natriuretic peptide is helpful in ruling out the condition. In a study involving a broad range of patients presenting to the emergency department with shortness of breath, a cutoff level of B-type natriuretic peptide of $100 \mathrm{pg} / \mathrm{mL}$ produced a sensitivity of $90 \%$ ( $95 \%$ CI $88 \%-92 \%)$, a specificity of $76 \%$ ( $95 \% \mathrm{CI} 73 \%-79 \%$ ), a positive predictive value of $79 \%(95 \%$ CI $76 \%-8 \mathrm{r} \%)$ and a negative predictive value of $89 \%$ (95\% CI $87 \%-91 \%)$, compared with the "gold standard" of the final diagnosis being determined by a cardiologist. ${ }^{17}$ An elevated level of B-type natriuretic peptide may be difficult to interpret in patients with stable, compensated cardiac dysfunction, because they often have chronically elevated levels of this peptide. ${ }^{18}$ Assays for $\mathrm{N}$-terminal B-type natriuretic peptide have similar characteristics to those of assays for B-type natriuretic peptide, but the absolute values are roughly 6 -fold higher. Transthoracic echocardiography is useful in the diagnosis of acute decompensated heart failure

Box 1: Proposed causes of acute decompensated heart failure due to left ventricular dysfunction

Primary cardiac

- Progressive cardiomyopathy with remodelling

- Acute cardiomyopathy (myocarditis, postpartum cardiomyopathy)

- Myocardial ischemia

- Arrhythmia (tachy- or bradyarrhythmia)

- Valvular dysfunction (stenosis or regurgitation)

- Pericardial syndrome (tamponade, constriction)

Pressure overload

- Hypertensive urgency or emergency

Volume overload

- Sodium or volume load

- Decreased compliance with diuretics

- Renal dysfunction

- Hepatic dysfunction

High output

- Shunt (intra- or extracardiac)

- Anemia

- Septicemia

- Thyroid disease

Other

- Inflammation or infection

- Major surgery

- Lack of compliance with heart failure medications

- New medications (excess $\beta$-blockade)

- Substance abuse (alcohol, stimulants) through its ability to evaluate left ventricular systolic function, diastolic functional class, valvular function, left atrial filling pressures, right ventricular systolic pressure and inferior vena caval pressure. Electrocardiography, complete blood count, basic metabolic panel and possibly thyroid function tests are indicated in patients suspected of having acute decompensated heart failure. Pulmonary artery catheterization may provide helpful information about cardiac hemodynamics, particularly in cases of shock (Fig. I). Although a central venous pressure line or pulmonary artery catheter provides a good measure of filling pressures and the latter can estimate cardiac output, neither has been shown to improve outcomes when used in acute decompensated heart failure. ${ }^{9}$

\section{Treatment}

The last 2 decades have seen the successful development of a number of therapies for chronic systolic dysfunction. Angiotensin-converting-enzyme (ACE) inhibitors, ${ }^{19,20}$ angiotensin II receptor blockers, ${ }^{21,22} \beta$-blockers, ${ }^{23,24}$ aldosterone antagonists ${ }^{25}$ implantable cardioverter defibrillators ${ }^{26}$ and cardiac resynchronization therapy ${ }^{27}$ have all been shown in large, prospective, randomized controlled trials to reduce morbidity and mortality among patients with stable congestive heart failure and reduced left ventricular ejection fraction. Unfortunately, the same success has not been seen in the treatment of acute decompensated heart failure. Consequently, therapy for acute decompensated heart failure has not changed significantly over the past 30 years (Table $\mathrm{I}$ ), ${ }^{28}$ and neither has the risk of death among patients admitted to hospital with the condition (30-day mortality I $2 \%$ and I-year mortality $28 \%$ during $1950-1969$, and $\mathrm{II} \%$ and $28 \%$ respectively during 1990-I999). ${ }^{29}$ Fig. 2 provides a summary schematic of short-term therapies that may be used to stabilize the condition of patients presenting with acute decompensated heart failure.

Box 2: Findings in patients with suspected acute decompensated heart failure

- Prior history of heart failure or myocardial injury

- Dyspnea on exertion, orthopnea or paroxysmal nocturnal dyspnea

- Fatigue

- Increasing edema, weight or abdominal girth

Physical examination

- Elevated jugular venous pressure

- Peripheral edema or ascites

- Rales, hypoxia or tachypnea

- Tachycardia, arrhythmia

- Diffuse point of maximal intensity

- Ventricular filling gallop (S3)

- Atrial gallop (S4)

- Cool extremities above the hands and feet

- Poor urine output 


\section{Loop diuretics}

Volume overload is central to the pathophysiology of most episodes of acute decompensated heart failure. Elevated filling pressures are ultimately responsible for many of the signs and symptoms of heart failure. Consequently, the goals of care, particularly in the acute setting, include the relief of congestion. Most patients presenting with severe acute decompensated heart failure have a pulmonary capillary wedge pressure greater than $25 \mathrm{~mm} \mathrm{Hg.}{ }^{9}$ In addition, patients whose left and right filling pressures can be reduced during their hospital stay typically experience alleviation of symptoms. ${ }^{30} \mathrm{~Pa}-$ tients whose B-type natriuretic peptide level decreases substantially between admission and discharge have a much reduced likelihood of repeat hospital admission. ${ }^{18}$

As a reflection of the critical role of congestion in acute decompensated heart failure, clinicians rely heavily on diuretic therapy. Of patients with acute decompensated heart failure followed in recent large registries, 90\% received loop diuretics, with $70 \%$ receiving it as monotherapy; nitroglycerin and nesiritide (natriuretic peptide, not available in Canada) were the next most commonly prescribed therapies, each used in only 10\% of patients. ${ }^{31}$ Loop diuretics are the mainstay of diuretic therapy because they produce significantly more natriuresis than other diuretics, particularly in the setting of decreased glomerular filtration rates. There is some evidence that, when high doses of intravenous furosemide are required, continuous infusion may have benefits over bolus dosing. ${ }^{32}$ The non-loop diuretics can complement loop diuretic therapy: for example, metolazone to potentiate natriuresis, spirinolactone to stem potassium losses, and acetazolamide to correct hypochloremic metabolic acidosis.

In a meta-analysis of randomized controlled trials of diuretics in outpatients with congestive heart failure, the 3 placebo-controlled trials reporting mortality data showed that the rate of death was lower among patients given diuretics than among those given placebo $(n=22 \mathrm{I}$, odds ratio 0.25 , 95\% CI 0.07-0.84). ${ }^{33}$ However, as should be recognized throughout this discussion, therapies for stable congestive heart failure may not be as effective when used to treat acute decompensated heart failure.

Despite these seemingly clear clinical indications for loop diuretics in the reduction of volume overload, there is controversy regarding their routine use in acute decompensated heart failure. No large prospective trial has ever looked at diuretics in acute decompensated heart failure, in part because these drugs are central to the treatment of patients with evidence of volume overload. However, most observational studies involving patients with acute decompensated heart failure have shown strong associations between increasing diuretic dose and worsening mortality, even after controlling for multiple covariates. ${ }^{31,34,35}$ Certainly, increasing diuretic dose is a marker of disease severity. However, there are reasons to suspect that aggressive use of loop diuretics may play a causal role in worsened outcomes. Loop diuretics typically decrease renal perfusion and glomerular filtration rates, which promotes kidney dysfunction. They also worsen neurohormonal activation, which stimulates the renin-angiotensin-aldosterone axis. In a
Box 3: Tests that may help in the diagnosis and treatment of acute decompensated heart failure

- Chest radiography

- Electrocardiography

- Measurement of B-type natriuretic peptide and $\mathrm{N}$-terminal B-type natriuretic peptide levels

- Other laboratory tests (complete blood count, renal function tests, measurement of electrolyte levels, glucose level, transaminase levels, prothrombin time, troponin level, D-dimer level and arterial blood gas pressure, thyroid function tests and urinalysis)

- Transthoracic echocardiography

- Central venous line or pulmonary artery catheter

study of the hemodynamic and neurohumoral responses to acute diuretic therapy in 15 patients with severe congestive heart failure, a bolus of furosemide given intravenously acted as an arteriolar vasoconstrictor within the first hour after administration..$^{36}$ Loop diuretics can exacerbate electrolyte abnormalities, primarily causing hypokalemia, particularly when used in combination with thiazides.

A small randomized study involving patients presenting to the emergency department with acute decompensated heart failure and respiratory distress compared high-dose isosorbide dinitrate (3-mg bolus every 5 minutes) with high-dose furosemide (8o-mg bolus every 15 minutes) plus low-dose isosorbide dinitrate ( $\mathrm{m} \mathrm{mg} / \mathrm{h}$ doubled every Io minutes). All patients initially received oxygen therapy and a $40-\mathrm{mg}$ bolus of furosemide before being randomly assigned to the treatment groups; treatment was continued until oxygen saturation was $96 \%$ or the mean arterial pressure decreased by $30 \%$ or to below $90 \mathrm{~mm} \mathrm{Hg}$. Among the ro4 patients enrolled, mechanical ventilation was required by more patients in the high-dose furosemide group than in the high-dose isosorbide dinitrate group ( $40 \%$ v. $13 \%, p<0.004) .^{37}$

Because of concerns about loop diuretics in the acute setting and because of the increasing prevalence of diureticresistant patients, there is increasing interest in alternative approaches to diuresis. However, until newer therapies are proven to be superior to loop diuretics in a wide range of patients with acute decompensated heart failure, furosemide will continue to be the mainstay of therapy. Given the existing data, we recommend the use of intravenous furosemide therapy in patients with evidence of volume overload and adequate blood pressure, possibly as a continuous infusion when doses greater than $80 \mathrm{mg} / \mathrm{d}$ are required. However, loop diuretics should typically be used in combination with vasoand venodilator therapies, with careful monitoring to avoid overdiuresis and resulting hypotension or renal dysfunction.

\section{Ultrafiltration}

Peripheral ultrafiltration may be the most promising new treatment option for patients with acute decompensated heart failure. Small clinical trials comparing intermittent peripheral 
venovenous ultrafiltration with intravenous diuretic therapy in patients with acute decompensated heart failure who had volume overload showed greater volume removal, greater decrease in B-type natriuretic peptide levels and shorter lengths of stay among those receiving the ultrafiltration. ${ }^{38,39}$ The Ultrafiltration versus Intravenous Diuretics for Patients Hospitalized for Acute Decompensated Heart Failure (UNLOAD) trial involved 200 patients with acute decompensated heart failure and showed that peripheral ultrafiltration compared with diuretics alone improved weight loss at 48 hours $(5.0 \mathrm{~V} .3 . \mathrm{rgg}$, $p<0.00 \mathrm{I})$, decreased the need for vasoactive drugs $(3 \% \mathrm{v}$. $13 \%, p=0.02)$ and reduced the rate of readmission to hospital at 90 days ( $18 \%$ v. $32 \%, p=0.02$ ). ${ }^{40}$ Mechanisms behind these relative benefits are yet to be determined. The up-front cost of peripheral ultrafiltration is relatively expensive, but it could possibly be defrayed by cost savings down the road. Although ultrafiltration has recently been approved in the United States for the treatment of volume overload, whether it will become routinely used in the general care of patients with acute decompensated heart failure remains to be determined.

\section{Vasodilators}

Preload reduction with venodilators is thought to be helpful in acute decompensated heart failure by reducing congestion and minimizing cardiac oxygen demand. Intravenous nitrate therapy resulted in acute improvement of dyspnea in 2 randomized trials involving patients with severe acute decompensated heart failure. ${ }^{37,41}$ Similarly, morphine acts as a venodilator and mild arterial dilator, and it centrally suppresses symptoms of breathlessness. However, no rigorous studies of morphine have been performed in acute decompensated heart failure.

Afterload reduction is also thought to be helpful in some patients with acute decompensated heart failure by decreasing myocardial oxygen demand and improving forward flow. The majority of patients with acute decompensated heart failure have systemic hypertension at presentation. ${ }^{12}$ The use of a short-acting arterial-dilating agent, such as nitroprusside, tends to be preferred in patients with acute decompensated heart failure who have borderline blood pressure, and it may benefit patients with persistent pump failure of more than 9 hours' duration after acute myocardial infarction. ${ }^{42}$ Transition to an ACE inhibitor or an angiotensin II receptor blocker is recommended as soon as renal function and blood pressure allow. The combined use of hydralazine and isosorbide dinitrate is an alternative in patients who are black or who have significant renal dysfunction. ${ }^{43,44}$ These agents not only have theoretical benefits in acute decompensated heart failure but are also central to the long-term treatment of underlying chronic systolic dysfunction. ${ }^{19-22}$

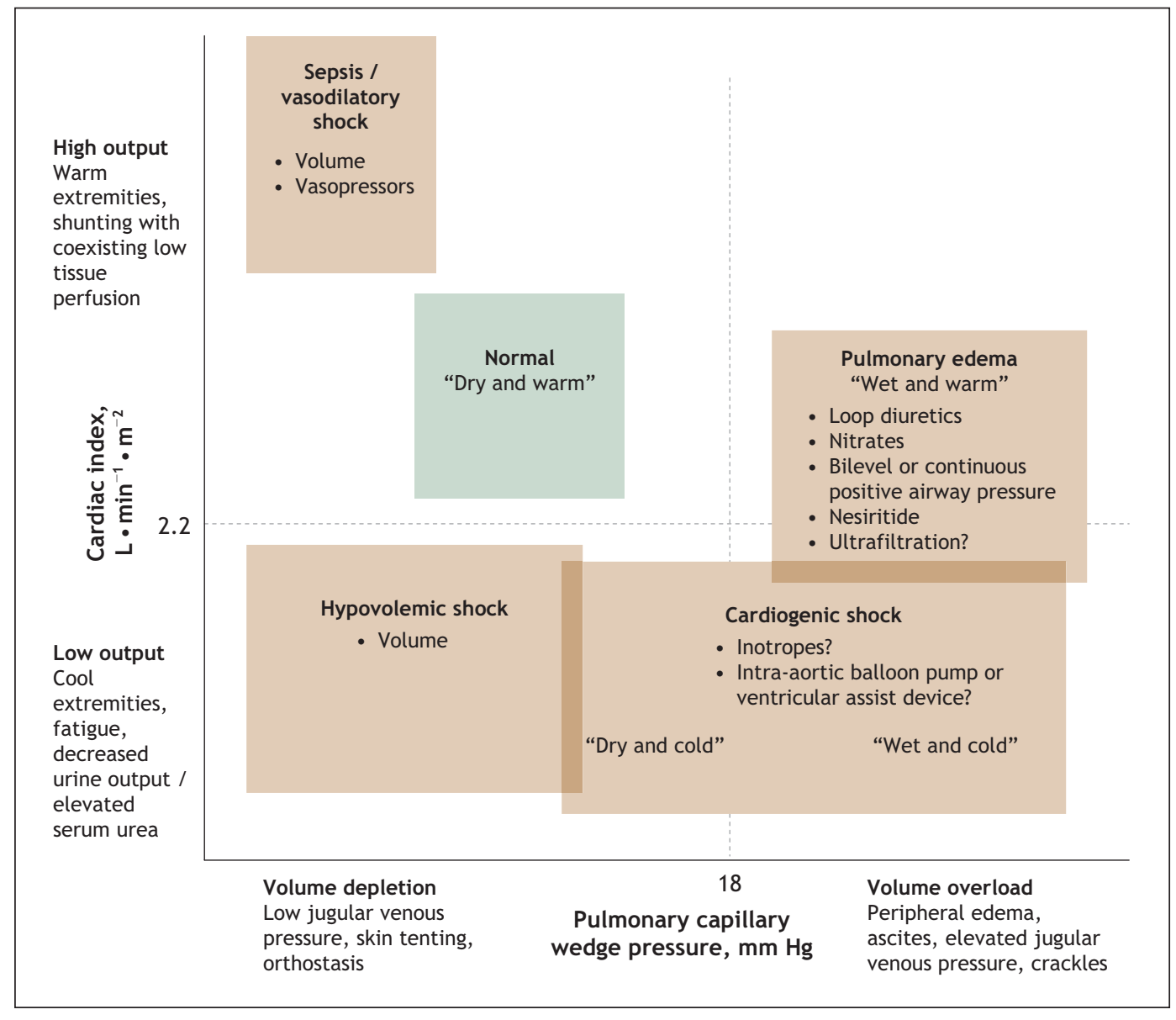

Fig. 1: Approximation of cardiac systolic function and cardiac filling pressures in various acute illnesses. ${ }^{30}$ 
Table 1: Summary of selected treatment options for acute decompensated heart failure

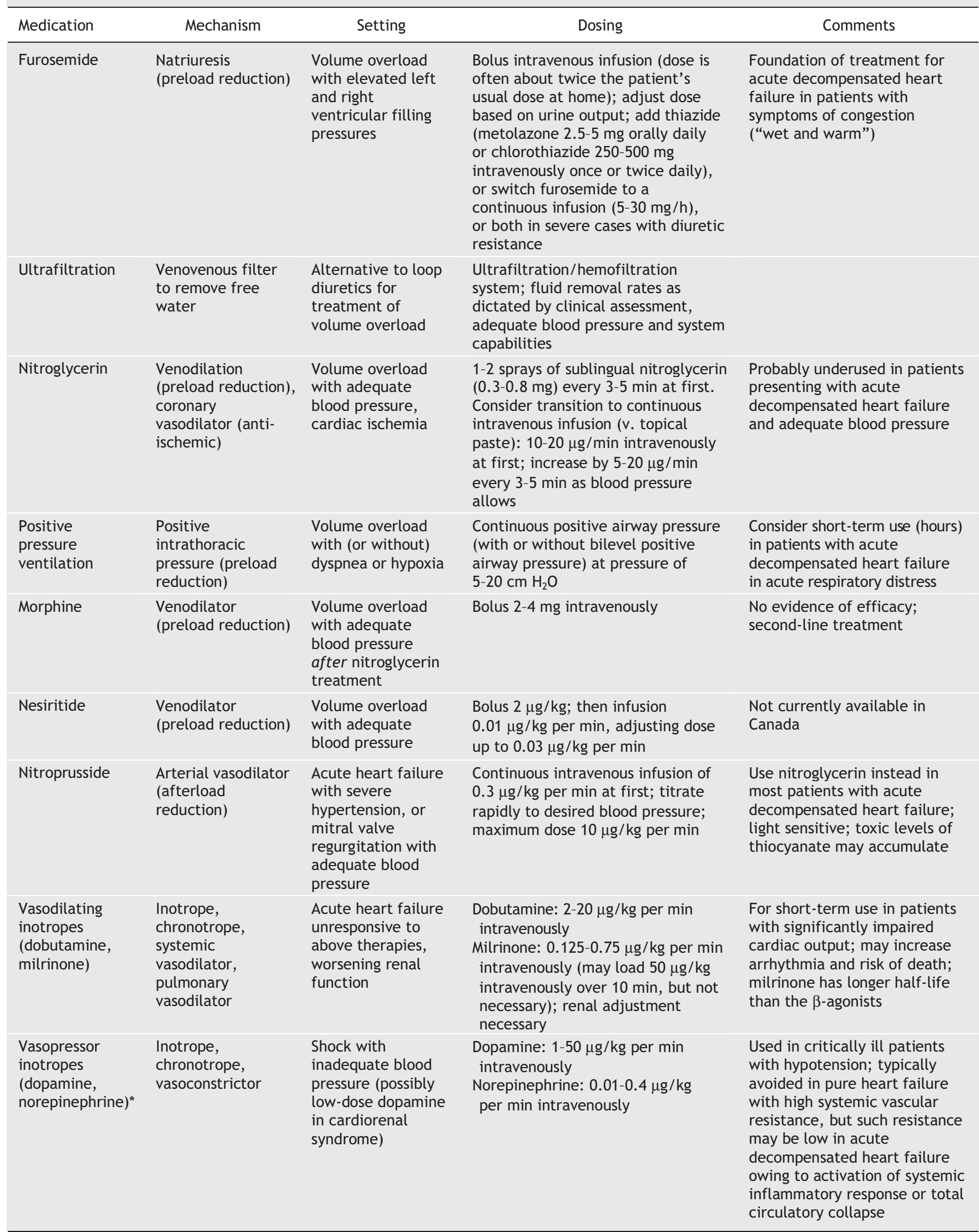




\section{Natriuretic peptides}

Natriuretic peptides have been dubbed counter-regulatory hormones because their endogenous production is in response to atrial and ventricular stretch, purportedly acting to offset the harmful spiral of sodium retention and vasoconstriction central to the pathophysiology of acute decompensated heart failure. Although the vasodilatory properties of natriuretic peptides have been well validated, the natriuretic effects at recommended doses have been modest in clinical trials. Nesiritide (recombinant B-type natriuretic peptide), made available for clinical use in the United States in 200I but not currently available in Canada, was the first newly approved drug for the treatment of acute decompensated heart failure by the US Food and Drug Administration since 1987. Despite its initial rapid acceptance in the treatment of acute decompensated heart failure in many parts of the world, nesiritide has recently come under scrutiny, and its future role in the treatment of this condition is likely to be determined through further study.

The largest study to date evaluating nesiritide is the Vasodilation in the Management of Acute Congestive Heart Failure (VMAC) trial. ${ }^{41}$ The trial randomly assigned 498 patients with New York Heart Association class IV heart failure requiring hospital care for dyspnea to I of 3 treatment arms for 3 hours: nesiritide, adjustable dose up to $0.03 \mu \mathrm{g} / \mathrm{kg}$ per minute; intravenous nitroglycerin therapy, with the dosage determined at the investigator's discretion; or placebo. This was followed by another round of randomization of the placebo group such that, from hour 3 to hour 24, patients received a fixed dose of nesiritide, an adjustable dose of nesiritide or nitroglycerin.
The results demonstrated that nesiritide was the most effective in reducing the pulmonary capillary wedge pressure at 3 hours (nesiritide $-5.8 \mathrm{~mm} \mathrm{Hg}[p<0.00 \mathrm{I}$ v. placebo], nitroglycerin $-3.8 \mathrm{~mm} \mathrm{Hg}[p<0.09 \mathrm{v}$. placebo], placebo $-2.0 \mathrm{~mm} \mathrm{Hg}$ ); however, the difference was no longer significant at 48 hours. There was no significant difference in selfreported dyspnea or global clinical status at 3 and 24 hours between nesiritide and nitroglycerin. Nesiritide was superior to placebo in reducing dyspnea at 3 hours, but this effect waned over time such that the effect of nesiritide was not distinguishable from that of placebo on either dyspnea or global status at other future time points when compared with the 3hour placebo measures. Hypotension was more common in the nesiritide group. The 30-day readmission rate was $20 \%$ among the patients given nesiritide and $23 \%$ among those given nitroglycerin.

In a post-hoc meta-analysis of trial data submitted to the US Food and Drug Administration, nesiritide was found to increase the risk of renal dysfunction significantly when compared with placebo or active control (relative risk I.54; 95\% CI I.I9-I.98) ${ }^{45}$ Worsening renal function is known to correlate with worse outcomes in patients with acute decompensated heart failure. However, there was no clear statistical difference in the need for dialysis between the nesiritide group and the control group ( $2.5 \%$ v. $2.2 \%, p=0.7 \mathrm{I})$. Pooling of data from the 3 randomized trials of nesiritide versus noninotropic control that recorded 30-day survival (which also represented the only 3 existing trials of nesiritide focused on patients with acute decompensated heart failure) revealed a trend toward increased mortality among patients given nesiritide $(7.2 \%$ v. $4.0 \% ; p=0.059) .{ }^{46}$ In response to these con-

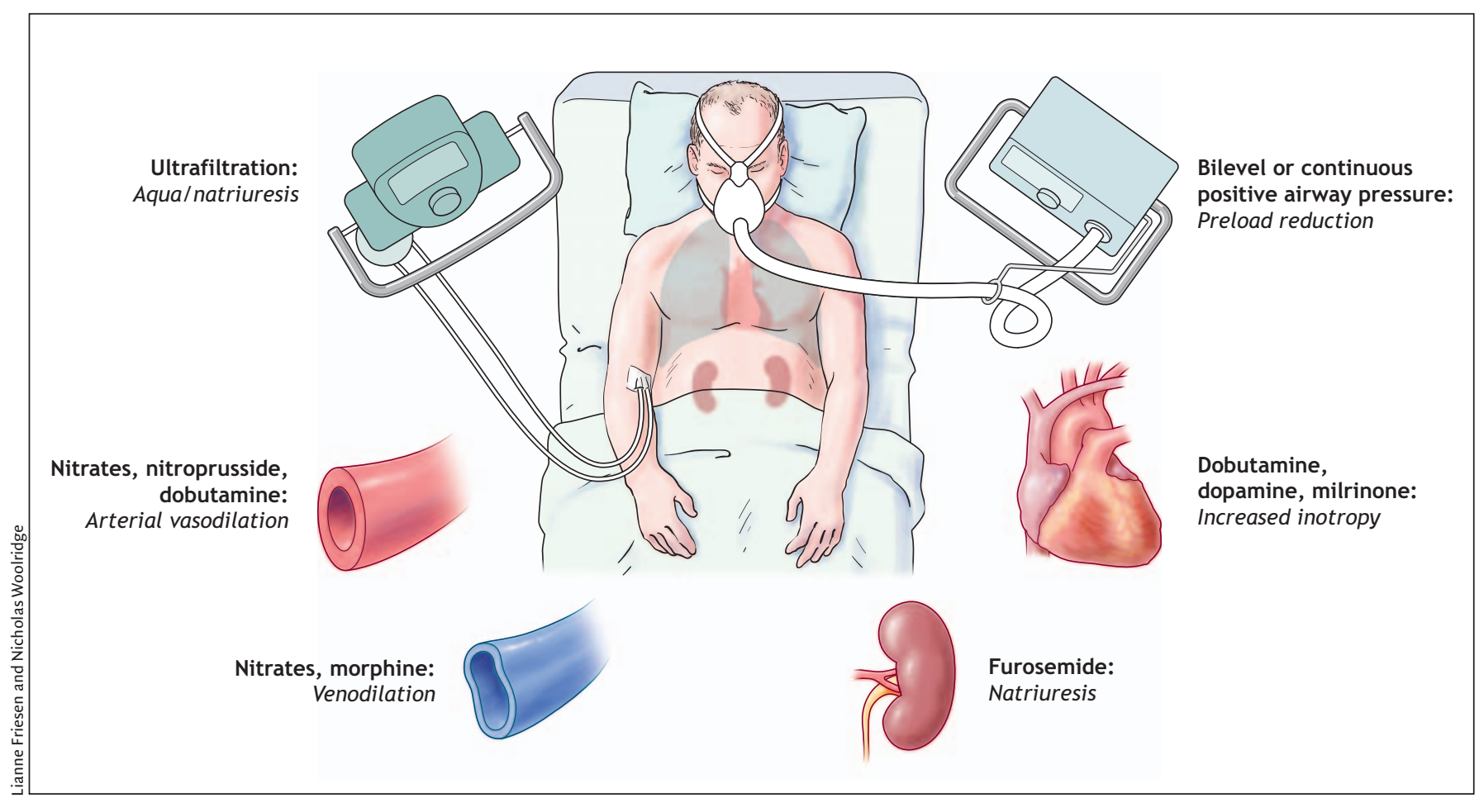

Fig. 2: Various targets for therapies used in the management of acute decompensated heart failure. 
cerns, the manufacturer of nesiritide recently announced plans to conduct a randomized controlled trial involving up to 7000 patients with acute decompensated heart failure starting in 2007 that will evaluate morbidity and mortality as well as renal dysfunction, quality of life and cost-effectiveness.

\section{Respiratory therapies}

In patients with pulmonary edema and hypoxia, the use of supplemental oxygen is recommended. Short-term positive pressure ventilation should be considered first-line treatment of acute cardiogenic pulmonary edema. This nonpharmacologic therapy is thought to act primarily by decreasing venous return to the heart. In a meta-analysis of multiple small trials, noninvasive continuous positive airway pressure or CPAP was found to clearly reduce the need for mechanical ventilation (relative risk $0.44,95 \% \mathrm{CI} 0.29-0.66$ ) and was associated with decreased mortality (relative risk $0.59,95 \%$ CI $0.3^{8-}$ 0.90)..$^{47,48}$ In the setting of acute decompensated heart failure, bilevel positive airway pressure or BiPAP is probably equivalent to continuous positive airway pressure. In a study involving patients with chronic heart failure who had central sleep apnea, continuous positive airway pressure improved many secondary outcome measures but did not significantly improve transplant-free survival at 18 months (hazard ratio 0.66 , $p=0.06$ ), such that extended use of continuous positive airway pressure is of questionable benefit. ${ }^{49}$ Noninvasive continuous positive pressure ventilation is not proven in acute myocardial infarction with respiratory distress. Therefore, invasive mechanical ventilation, with placement of an endotracheal tube, is probably more appropriate in such situations, particularly if the patient needs to be taken urgently to the catheterization laboratory.

\section{Inotropes}

Short-term inotropic infusion, although frequently used to improve hemodynamics and symptoms in acute decompensated heart failure, remains controversial. When patients present with profound circulatory collapse, inotropes may be absolutely required. For patients with acute decompensated heart failure who have evidence of end-organ hypoperfusion or diuretic resistance, but no frank hypotension, the use of inotropes is not well supported.

Dobutamine is a synthetic catecholamine with mainly $\beta_{1^{-}}$ receptor agonism and some $\beta_{2}$-receptor activity, characteristics that make it an inotropic vasodilator. Use of milrinone, a phosphodiesterase III inhibitor, results in elevated levels of cyclic adenosine monophosphate in the myocardium and smooth muscle, which leads to increased cardiac contractility and vasodilation. Milrinone produces hemodynamic changes similar to those of dobutamine, but because it works via a different cellular signaling pathway, it can be used simultaneously with catecholaminergic agonists or antagonists.

The use of dobutamine for inotropic support in acute decompensated heart failure has yielded mixed results in studies. A single infusion of dobutamine for $3^{-5}$ days in patients with acute decompensated heart failure has been shown to improve symptoms for up to 30 days..$^{50,51}$ Intermittent dobutamine therapy in ambulatory patients with severe chronic heart failure showed a nonsignificant trend toward worse outcomes. ${ }^{52}$ The largest registry of patients with acute decompensated heart failure to date associated higher mortality with intravenous inotrope therapy than with nitroglycerin or nesiritide therapy. ${ }^{53}$ The use of digoxin has been shown to reduce rates of hospital admission among patients with chronic heart failure without a significant effect on mortality, ${ }^{54}$ but its use in acute decompensated heart failure is not defined beyond small studies looking at hemodynamic effects. Longterm digoxin therapy should not be stopped during acute decompensated heart failure, because this may worsen cardiac function in the short term.

The Outcomes of a Prospective Trial of Intravenous Milrinone for Exacerbations of Chronic Heart Failure (OPTIMECHF) enrolled 95I patients who were admitted with hemodynamically stable exacerbations of systolic heart failure. ${ }^{8}$ Patients with evidence of frank cardiogenic shock were excluded. Within the first 48 hours after admission, patients were randomly assigned to receive either a $48-72$-hour infusion of milrinone (initially $0.5 \mu \mathrm{g} / \mathrm{kg}$ per min) or placebo. No statistical difference was found between the 2 arms at 60 days for the primary end point of total days in hospital (mean 6 days in the milrinone group v. 7 days in the placebo group; $p=0.7 \mathrm{I})$. In addition, mortality did not differ significantly between the 2 groups, although it tended to be higher in the milrinone group than in the placebo group (in-hospital mortality $3.8 \%$ v. $2.3 \%$ respectively; $p=0.19$ ). Multiple secondary clinical end points were significantly worse in the milrinone group, including sustained hypotension requiring intervention (I0.7\% v. $3.2 \% ; p<0.001$ ) and new atrial arrhythmias $(4.6 \%$ v. I. $5 \% ; p=0.004)$.

Despite these fairly robust negative findings, milrinone, dobutamine and dopamine continue to be used relatively frequently in the management of acute decompensated heart failure, especially when more conservative therapies fail. Generalizability is always a problem with trials; there may be subgroups of patients with acute decompensated heart failure who could benefit from inotropic support. However, the preponderance of evidence does not bear this out, and consequently $\beta$-agonists and phoshodiesterase inhibitors should typically be avoided or limited to short-term or palliative use.

Because of concern about the effect of abrupt withdrawal of $\beta$-blocker therapy in acute decompensated heart failure, use of a phosphodiesterase inhibitor in combination with continued or tapered $\beta$-blocker therapy may, in theory, provide benefit over $\beta$-agonist therapy. ${ }^{55}$ What to do with longterm $\beta$-blocker therapy in the setting of acute decompensated heart failure remains a clinical conundrum. Our practice is to reduce the dose proportionate to the degree of hemodynamic compromise; the $\beta$-blocker dose may be decreased by about half in patients with evidence of hypoperfusion, and stopped in patients with frank cardiogenic shock, although there is little evidence to support this approach. Following an episode of acute decompensated heart failure, $\beta$-blocker therapy should be titrated upward slowly. 


\section{Future targets}

Vasopressin receptor antagonists (tolvaptan and conivaptan), ${ }^{56,57}$ calcium sensitizers (levosimendan) ${ }^{58,59}$ and endothelin receptor antagonists (tezosentan and bosentan) ${ }^{60-62}$ represent some of the potential therapeutic avenues that have been investigated in recent years. Although levosimendan is currently available in Europe, none of these agents is currently in widespread use owing to relatively neutral findings in clinical trials to date. Encouraging data from early studies have indicated that adenosine $A_{1}$ receptor antagonists show promising diuretic properties in patients with acute decompensated heart failure, particularly diuretic-refractory patients; phase III trials are now underway. New inotropes that enhance the efficiency of actin-myosin coupling and minimally invasive mechanical ventricular support devices are some of the technologies in early development that may ultimately change the landscape of management of acute decompensated heart failure.

\section{Summary}

Acute decompensated heart failure is a common disease associated with major morbidity and mortality. Prompt recognition and appropriate treatment are important. Unfortunately, treatment options remain limited. Evidence of meaningful clinical outcomes with older therapies for acute decompensated heart failure is often lacking, and what data do exist frequently show marginal or mixed results. Intravenous therapy with a loop diuretic currently forms the foundation of care for patients with acute decompensated heart failure who have volume overload, but even furosemide use in acute decompensated heart failure is controversial. In patients with adequate blood pressure, vasodilators such as nitroglycerin are probably underused as an adjunct to diuresis. Short-term use of positive airway pressure in patients presenting with cardiogenic pulmonary edema may provide additional benefit. The initial goal is to stabilize the patient's condition with conservative measures that improve patient comfort, avoid mechanical ventilatory support, limit cardiac ischemia and attenuate the need for hospital admission. Once the patient's condition is stable, known therapies for congestive heart failure can then be established or reinstated. Patients with acute decompensated heart failure who present primarily with low cardiac output and renal dysfunction pose a more difficult clinical challenge. Inotropes have traditionally been used in such cases but have consistently failed to show benefit in clinical trials. Complex cases of heart failure with progressive renal dysfunction or persistent hypotension may require more aggressive therapies such as ventricular support (intra-aortic balloon counterpulsation or left-ventricular assist device), dialysis and heart transplantation - versus hospice - and thus should be managed by a specialist. Recently developed pharmaceutical agents have shown some promise in small studies, but those tested in larger randomized controlled trials have largely failed to show significant clinical benefit over older therapies. This area of medicine demands commitment to current and future clinical trials of both new and older treatment strategies. New technologies are being developed, and we can only hope that they will one day significantly enhance our ability to treat this difficult disease process.

\section{This article has been peer reviewed.}

From the Division of Cardiology, Department of Medicine, Duke University Medical Center, Durham, NC

Competing interests: None declared for Larry Allen. Christopher O'Connor has served as a consultant to, and has received research grants and honoraria from, Actelion (affiliated with tezosentan), Amgen, Astra, Bristol-Myers Squibb, GlaxoSmithKline, Guidant, Medtronic, Merck, Novartis, Otsuka America, Pfizer, and Scios (affiliated with nesiritide).

Contributors: Both authors contributed substantially to the design, analysis and writing necessary to produce this manuscript. Both approved the final version for publication.

\section{REFERENCES}

I. Nieminen MS, Bohm M, Cowie MR, et al; ESC Committee for Practice Guidelines (CPG). Executive summary of the guidelines on the diagnosis and treatment of acute heart failure: the Task Force on Acute Heart Failure of the European Society of Cardiology. Eur Heart J 2005;26:384-4I6.

2. Heart Failure Society of America. HFSA 2006 comprehensive heart failure practice guideline. Section 12: Evaluation and management of patients with acute decompensated heart failure. JCard Fail 2006; 12:e86-103.

3. Hunt SA, Abraham WT, Chin MH, et al. ACC/AHA 2005 guideline update for the diagnosis and management of chronic heart failure in the adult: a report of the American College of Cardiology/American Heart Association Task Force on Practice Guidelines (Writing Committee to Update the 200I Guidelines for the Evaluation and Management of Heart Failure). American College of Cardiology Web Site. Available: www.acc .org/qualityandscience/clinical/guidelines/failure/index.pdf (accessed 2007 Jan 3I).

4. Remme WJ, Swedberg K. Task Force for the Diagnosis and Treatment of Chronic Heart Failure, European Society of Cardiology. Guidelines for the diagnosis and treatment of chronic heart failure. Eur Heart J 2001;22:1527-60.

5. Braunwald E. Shattuck lecture - cardiovascular medicine at the turn of the millennium: triumphs, concerns, and opportunities. NEngl J Med 1997;337:1360-9.

6. Redfield MM. Heart failure - an epidemic of uncertain proportions. N Engl J Med 2002;347:1442-4.

7. McCullough PA, Philbin EF, Spertus JA, et al. Confirmation of a heart failure epidemic: findings from the Resource Utilization Among Congestive Heart Failure (REACH) study. J Am Coll Cardiol 2002;39:60-9.

8. Cuffe MS, Califf RM, Adams KF Jr, et al. Outcomes of a Prospective Trial of Intravenous Milrinone for Exacerbations of Chronic Heart Failure (OPTIME-CHF) Investigators. Short-term intravenous milrinone for acute exacerbation of chronic heart failure: a randomized controlled trial. JAMA 2002;287:I54I-7.

9. Binanay C, Califf RM, Hasselblad V, et al. ESCAPE Investigators and ESCAPE Study Coordinators. Evaluation study of congestive heart failure and pulmonary artery catheterization effectiveness: the ESCAPE trial. JAMA 2005;294:1625-33.

Io. Felker GM, Adams KF Jr., Konstam MA, et al. The problem of decompensated heart failure: nomenclature, classification, and risk stratification. Am HeartJ 2003 ; I45(2 Suppl):Sr8-25.

II. Mann DL. Mechanisms and models in heart failure: a combinatorial approach. Circulation 1999;100:999-1008.

I2. Adams KF Jr, Fonarow GC, Emerman CL, et al; ADHERE Scientific Advisory Committee and Investigators. Characteristics and outcomes of patients hospitalized for heart failure in the United States: rationale, design, and preliminary observations from the first Ioo,000 cases in the Acute Decompensated Heart Failure National Registry (ADHERE). Am Heart J 2005;I49:209-I6.

I3. Bales AC, Sorrentino MJ. Causes of congestive heart failure: Prompt diagnosis may affect prognosis. Postgrad Med I997; I0I:44-6.

I4. Wuerz RC, Meador SA. Effects of prehospital medications on mortality and length of stay in congestive heart failure. Ann Emerg Med I992;2I:669-74.

15. Badgett RG, Lucey CR, Mulrow CD. Can the clinical examination diagnose leftsided heart failure in adults? JAMA I997;277:1712-9.

I6. Wang CS, Fitzgerald JM, Schulzer M, et al. Does this dyspneic patient in the emergency department have congestive heart failure. JAMA 2005;294:1944-56.

I7. Maisel AS, Krishnaswamy P, Nowak RM, et al; Breathing Not Properly Multinational Study Investigators. Rapid measurement of B-type natriuretic peptide in the emergency diagnosis of heart failure. N Engl J Med 2002;347:I6I-7.

I8. Silver MA, Maisel A, Yancy CW, et al; BNP Consensus Panel. BNP Consensus Panel 2004. A clinical approach for the diagnostic, prognostic, screening, treatment monitoring, and therapeutic roles of natriuretic peptides in cardiovascular diseases. Congest Heart Fail 2004; Io(Suppl 3):I-30.

I9. SOLVD Investigators. Effect of enalapril on survival in patients with reduced left ventricular ejection fractions and congestive heart failure. $N$ Engl J Med I99I;325: 293-302. 
20. Cohn JN, Johnson G, Ziesche S, et al. A comparison of enalapril with hydralazineisosorbide dinitrate in the treatment of chronic congestive heart failure. $\mathrm{N} \mathrm{Engl} \mathrm{J}$ Med I991;325:303-I0.

2I. Cohn JN, Tognoni G; Valsartan Heart Failure Trial Investigators. A randomized trial of the angiotensin-receptor blocker valsartan in chronic heart failure. $N$ EngI $J$ Med 200I;345:1667-75.

22. McMurray J, Ostergren J, Swedberg K, et al; CHARM Investigators and Committees. Effects of candesartan in patients with chronic heart failure and reduced leftventricular systolic function taking angiotensin-converting enzyme inhibitors: the CHARM-Added trial. Lancet 2003;362:767-7I

23. Hjalmarson A, Goldstein S, Fagerberg B, et al; MERIT-HF Study Group. Effects of controlled-release metoprolol on total mortality, hospitalizations, and well-being in patients with heart failure: the Metoprolol CR/XL Randomized Intervention Trial in congestive Heart Failure (MERIT-HF). JAMA 2000;283:1295-302.

24. Packer M, Bristow MR, Cohn JN, et al; U.S. Carvedilol Heart Failure Study Group. The effect of carvedilol on morbidity and mortality in patients with chronic heart failure. N Engl J Med I996;334:1349-55.

25. Pitt B, Zannad F, Remme WJ, et al; Randomized Aldactone Evaluation Study (RALES) Investigators. The effect of spironolactone on morbidity and mortality in patients with severe heart failure. N Engl J Med I999;34I:709-I7.

26. Bardy GH, Lee KL, Mark DB, et al; Sudden Cardiac Death in Heart Failure Tria (SCD-HeFT) Investigators. Amiodarone or an implantable cardioverter-defibrillator for congestive heart failure. $N$ Engl J Med 2005;352:225-37

27. Cleland JG, Daubert JC, Erdmann E, et al; Cardiac Resynchronization Heart Failure (CARE-HF) Study Investigators. The effect of cardiac resynchronization on morbidity and mortality in heart failure. N Engl J Med 2005;352:1539-49.

28. Ramirez A, Abelmann WH. Cardiac decompensation. N Engl J Med i974;290: 499-501.

29. Levy D, Kenchaiah S, Larson MG, et al; Framingham Heart Study. Long-term trends in the incidence of and survival with heart failure. N Engl J Med 2002;347 I397-402.

30. Nohria A, Mielniczuk LM, Stevenson LW. Evaluation and monitoring of patients with acute heart failure syndromes. Am J Cardiol 2005;96(6A):32G-40G.

3I. Emerman CL, Marco TD, Costanzo MR, et al. Impact of intravenous diuretics on the outcomes of patients hospitalized with acute decompensated heart failure: insights from the ADHERE registry [abstract]. J Card Fail 2004;I0:Sir6.

32. Salvador DR, Rey NR, Ramos GC, et al. Continuous infusion versus bolus injection of loop diuretics in congestive heart failure. Cochrane Database Syst Rev 2005(3): $\mathrm{CDoO} 3178$.

33. Faris R, Flather M, Purcell H, et al. Current evidence supporting the role of diuretics in heart failure: a meta analysis of randomised controlled trials. Int J Cardio 2002;82:149-58.

34. Neuberg GW, Miller AB, O'Connor CM, et al; PRAISE Investigators. Diuretic resistance predicts mortality in patients with advanced heart failure. Am Heart $J$ 2002;I44:3 I-8.

35. Domanski M, Norman J, Pitt B, et al. Studies of Left Ventricular Dysfunction. Diuretic use, progressive heart failure, and death in patients in the Studies Of Left Ventricular Dysfunction (SOLVD). J Am Coll Cardiol 2003;42:705-8.

36. Francis GS, Siegel RM, Goldsmith SR, et al. Acute vasoconstrictor response to intravenous furosemide in patients with chronic congestive heart failure: activation of the neurohumoral axis. Ann Intern Med ig85; I03:I-6

37. Cotter G, Metzkor E, Kaluski E, et al. Randomised trial of high-dose isosorbide dinitrate plus low-dose furosemide versus high-dose furosemide plus low-dose isosorbide dinitrate in severe pulmonary oedema. Lancet I998;351:389-93.

38. Jaski BE, Ha J, Denys BG, et al. Peripherally inserted veno-venous ultrafiltration for rapid treatment of volume overloaded patients. J Card Fail 2003;9:227-3I.

39. Bart BA, Boyle A, Bank AJ, et al. Ultrafiltration versus usual care for hospitalized patients with heart failure: the Relief for Acutely Fluid-Overloaded Patients With Decompensated Congestive Heart Failure (RAPID-CHF) trial. J Am Coll Cardiol 2005;46:2043-6.

40. Costanzo MR, Guglin ME, Saltzberg MT, et al; UNLOAD Trial Investigators. Ultrafiltration versus intravenous diuretics for patients hospitalized for acute decompensated heart failure. J Am Coll Cardiol 2007;49:675-83.

4I. Publication Committee for the VMAC Investigators (Vasodilatation in the Manage ment of Acute CHF). Intravenous nesiritide versus nitroglycerin for treatment of decompensated congestive heart failure: a randomized controlled trial. JAMA 2002;287:I53I-40.

42. Cohn JN, Franciosa JA, Francis GS, et al. Effect of short-term infusion of sodium nitroprusside on mortality rate in acute myocardial infarction complicated by left ventricular failure: results of a Veterans Administration cooperative study. $\mathrm{N} \mathrm{EngI}$ Med I982;306:II29-35.
43. Taylor AL, Ziesche S, Yancy C, et al; African-American Heart Failure Trial. (A-HeFT) Investigators. Combination of isosorbide dinitrate and hydralazine in blacks with heart failure. N Engl JMed 2004;35I:2049-57.

44. Cohn JN, Archibald DG, Ziesche S, et al. Effect of vasodilator therapy on mortality in chronic congestive heart failure: results of a Veterans Administration Cooperative Study (V-HeFT I). N Engl J Med I986;314:1547-52.

45. Sackner-Bernstein JD, Skopicki HA, Aaronson KD. Risk of worsening renal function with nesiritide in patient with acutely decompensated heart failure [published erratum in Circulation 2005;III:2274]. Circulation 2005;III:I487-91.

46. Sackner-Bernstein JD, Kowalski M, Fox M, et al. Short-term risk of death after treatment with nesiritide for decompensated heart failure: a pooled analysis of randomized controlled trials. JAMA 2005;293:1900-5.

47. Peter JV, Moran JL, Phillips-Hughes J, et al. Effect of non-invasive positive pressure ventilation on mortality in patients with acute cardiogenic pulmonary oedema: a meta-analysis. Lancet 2006;367:1155-63.

48. Winck JC, Azevedo LF, Costa-Pereira A, et al. Efficacy and safety of non-invasive ventilation in the treatment of acute cardiogenic pulmonary edema - a systematic review and meta-analysis. Crit Care 2006;I0:R69.

49. Bradley TD, Logan AG, Kimoff JR, et al; CANPAP Investigators. Continuous positive airway pressure for central sleep apnea and heart failure. N Engl J Med 2005; 353:2025-33.

50. Unverferth DV, Magorien RD, Lewis RP, et al. Long-term benefit of dobutamine in patients with congestive cardiomyopathy. Am Heart J I980;100:622-30.

5I. Liang CS, Sherman LG, Doherty JU, et al. Sustained improvement of cardiac function in patients with congestive heart failure after short-term infusion of dobutamine. Circulation I984;69:113-9.

52. Dies F, Krell MJ, Whitlow P. Intermittent dobutamine in ambulatory outpatients with chronic cardiac failure [abstract]. Circulation I986;74(Suppl II):38.

53. Abraham WT, Adams KF, Fonarow GC, et al; ADHERE Scientific Advisory Committee and Investigators. ADHERE Study Group. In-hospital mortality in patients with acute decompensated heart failure requiring intravenous vasoactive medications: an analysis from the Acute Decompensated Heart Failure National Registry (ADHERE). J Am Coll Cardiol 2005;46:57-64.

54. The Digitalis Investigation Group. The effect of digoxin on mortality and morbidity in patients with heart failure (the DIG trial). N Engl J Med I997;336:525-33.

55. Metra M, Dodari S, D'Aloia A, et al. Beta-blocker therapy influences the hemodynamic response to inotropic agents in patients with heart failure: a randomized comparison of dobutamine and enoximone before and after chronic treatment with metoprolol and carvedilol. J Am Coll Cardiol 2002;40:1248-58.

56. Gheorghiade M, Gattis WA, O'Connor CM, et al. Acute and Chronic Therapeutic Impact of a Vasopressin Antagonist in Congestive Heart Failure (ACTIV in CHF) Investigators. Effects of tolvaptan, a vasopressin antagonist, in patients hospitalized with worsening heart failure: a randomized controlled trial. JAMA 2004;29I:I963-7I.

57. Gheorghiade $M$, Gottlieb SS, Udelson JE, et al. Vasopressin $V_{2}$ receptor blockade with tolvaptan versus fluid restriction in the treatment of hyponatremia. Am J Cardiol 2006;97:1064-7.

58. Packer M. Randomized multicenter evaluation of intravenous levosimendan efficacy versus placebo in the short-term treatment of decompensated heart failure study (REVIVE-2). Proceedings of the American Heart Association Scientific Sessions; 2005 Nov I3-I6; Dallas.

59. Mebazaa A, Cohen-Solal A, Kleber F, et al. Study design of a mortality trial with intravenous levosimendan (the SURVIVE study) in patients with acutely decompensated heart failure. Crit Care 2004;8(Suppl I):87.

6o. Teerlink JR, Torre-Amione G. A new strategy for a clinical development program in acute decompensated heart failure: the randomized intravenous tezosentan (RITZ) trials [abstract]. J Card Fail 2000;69(Suppl 2):48.

6r. Teerlink JR, McMurray JJ, Bourge RC, et al; VERITAS Investigators. Tezosentan in patients with acute heart failure: design of the Value of Endothelin Receptor Inhibition with Tezosentan in Acute heart failure Study (VERITAS). Am Heart J 2005;150:46-53.

62. Kalra PR, Moon JC, Coats AJ. Do results of the ENABLE (Endothelin Antagonist Bosentan for Lowering Cardiac Events in Heart Failure) study spell the end for non-selective endothelin antagonism in heart failure? Int J Cariol 2002;85:195-7.

Correspondence to: Dr. Christopher M. O'Connor, Division of Cardiology, Department of Medicine, Duke University Medical Center, Box 3356, Durham NC 27710, USA; fax 9I9 68I-7755; christophe.oconnor@duke.edu 